\title{
Calibrated phasor measurement unit as a reliable metrological tool for national power grid operation in India
}

\author{
Saood Ahmad*, Anish M. Bhargav, Anurag Gupta, V. N. Ojha and D. K. Aswal \\ Indian Standard Time Division, CSIR-National Physical Laboratory, New Delhi 110 012, India
}

At the national power grid in India, stability is one of the most important factors due to disturbances caused by distributed load and time-variant sources. Presently, for monitoring the transmission efficiency and performance of power grids, phasor measurement units (PMUs) are being installed at various locations in the country. Time synchronization, using Coordinated Universal Time (UTC), makes PMU an important and reliable data acquisition equipment across the grids. To ensure reliability and accuracy of the acquired data, PMUs must be calibrated. However, recent development of automated PMU calibrator system by NIST and M/s Fluke, USA has revolutionized the calibration process by enhancing the accuracy and consistency of PMU measurements. The CSIR-NPL PMU system is fully operational to calibrate PMUs according to the IEEE synchrophasor standards. The time consumed to perform the PMU calibration is comparatively much less than the conventional method. A traceable PMU calibrator system has great potential in calibrating PMUs used to monitor, control and protect the power grid.

Keywords: Calibration, national power grid, phasor measurement unit, synchrophasor, time synchronization, uncertainty.

IN terms of electricity production and consumption, India stands at the third and fourth positions for the year 2017, as stated in a report published by India Brand Equity Foundation (IBEF) ${ }^{1}$. With advancements in industries and technology, the demand for power consumption has increased significantly in the country. In India, both renewable and non-renewable sources are used for power generation and further distribution. India's national power grid consists of five regional grids: eastern, northern, western, southern and northeastern. There is an interconnection among these grids achieved using high-capacity synchronous links. The rise in energy demand has led to the introduction of new technologies and concepts by the power generation and distribution entities. The prime focus is on monitoring the variations in voltage and current levels in realtime, effective distribution of load,

\footnotetext{
*For correspondence. (e-mail: ahmads@nplindia.org)
}

etc. The SCADA/EMS (Supervisory Control and Data Acquisition System/Energy Management System) set-up effectively studies steady-state effects occurring in grids. To analyse the transient effects in grids, synchrophasor measurements over a wide area are required ${ }^{2}$. PMU assists in performing these measurements and is used effectively at different power stations.

The URTDSM (Unified Real-Time Dynamic State Measurement) project of the Power Grid Corporation of India Limited (PGCIL) is the largest working project with the objective to monitor and control the supply of electricity throughout the country. The Central Transmission Utility (CTU) has proposed to install phasor measurement units (PMU) at all substations and power-generating stations. The power grid plan is to install PMUs at different locations on the national grid in different phases; more than 1000 PMUs have been installed so far. The PMU network can monitor the grid events such as flow of power, voltage, power demand in realtime and corresponding supply synchronization, etc. This leads to improvement in the stability of the grid, enhances reliability, causes a reduction in the possibility of blackouts and minimizes the impact of grid curtailment on the economy ${ }^{3}$. With the National Transmission Asset Management Centre (NTAMC), the grid is monitored by PGCIL at regional levels. NTAMC then further monitors the status of different locations through control centres.

The stability of the grid, which was better previously, had now been altered due to disturbances that occur on a day-to-day basis. It becomes essential to have a database of the power generated and distributed along with the transmission record. This will help in achieving control over the entire grid performance, which is also one of the critical requirements of the country. Around the world, the smart grid concept has gained momentum to tackle the current scenario as it helps control, monitor and analyse the power requirements, which in turn increase overall efficiency and stability. The Indian Government's ambitious plan, 'Power for All' needs additional power requirements. Still, it will face the challenges of overcoming a history of power quality, grid stability and frequent blackouts. Deployment of calibrated PMU at the power grid and monitoring the status is the only solution for all these above-mentioned issues. 


\section{Phasor measurement unit}

The sinusoidal wave function can be represented in terms of a complex number known as a phasor. The alternating current waveforms representing the signal in the power utility are defined in terms of the phasor and once they are time-stamped, they are known as synchrophasor. For time stamping, a global positioning system (GPS) is utilized $^{4}$. PMU measurements are taken at a very high speed compared to conventional technology. As of today, PMU has been accepted worldwide and is considered to be one of the critical elements in the power sector. It helps in analysing the conditions of the power system, assists in controlling and provides protection. PMU measures voltage, current and frequency of the transmission and distribution lines at the power grid using current transformers (CT) and potential transformers (PT) at very high speed. PMU assists in monitoring and enhancing the stability of the power grid. Calibrated PMUs give precision measurements necessary for minimizing and controlling power shutdowns and avoiding cascading blackouts, leading to economic growth of the country. Highly accurate and time-stamped PMU measurements recorded in different locations make nearly complete control of the entire power grid possible.

The synchrophasor PMU measurements result in a good indication of stress that exists on grids. With this information, it is possible to immediately apply any corrective action required to maintain the stability and reliability of the power grid. A PMU is a standalone device that can revolutionize the power sector if combined with other vital functions like relay protection or load shedding. In a particular region, PMUs are placed strategically at defined locations. The data from PMUs are processed at a common collection centre. Thus, it is possible to have complete power-related information about a particular region, such as power-supply details, fluctuations and disturbances, and its occurrence time, etc. The primary measurement and sensing capability of a PMU in a power grid makes it a tool well beyond just avoiding blackouts; for instance, by increasing line throughput and reducing line losses, the transmission and distribution efficiency of the grid can be improved. However, to accomplish this, PMUs need to be accurate, reliable and fully interchangeable, which is only possible by performing comprehensive and traceable PMU calibration. Such calibration requires the approved latest IEEE synchrophasor standards (C37.118.2011 and C37.118.1a-2014) compliance and the traceable PMU calibrator system along with its calibration procedure ${ }^{5}$.

\section{IEEE synchrophasor standards}

The Power Systems Relaying Committee (PSRC) of the IEEE Power Engineering Society developed a standard for the performance evaluation and calibration requirements of PMUs. The calibration aims to ensure that PMUs are accurate, reliable and fully interchangeable, and comply with the latest IEEE standards ${ }^{6}$. This standard for synchrophasor IEEE C37.118-2005 was published in December 2005, which specified the limits required for the PMUs. The standard explains the maximum allowable frequency error (FE), total vector error (TVE) under various operating conditions. These include frequency, voltage, current, phase angle and harmonic distortion. Now, the PSRC has ratified a new series of standards that updates and clarifies the existing standards as follows:

- IEEE C37.118.2011

- IEEE C37.118.1-2014

- IEEE C37.118.1a-2014

The accuracy and repeatability of PMUs according to specified IEEE standards are important to achieve the goal of the smart grid concept. CSIR-National Physical Laboratory (NPL), a National Metrological Institute of India (NMI), works towards developing and enhancing the calibration standards according to the country's need. The IEEE C37.118.1-2011 standard redefines the IEEE limits of PMU test acceptance (Table 1).

The latest standard for PMU evaluation (IEEE C37.118.1a-2014) includes improvements over the 2005 version and addendum in the 2011 version $^{7-10}$. The new version clearly explains the definitions of phasors and synchronized phasors. TVE and compliance tests exist as earlier, but have been expanded. The dynamic tests that help in understanding the performance have been introduced. Frequency and rate of change of frequency (ROCOF) measurement limits have been developed.

IEEE standard C37.118.1 comprises two main sections:

$>$ Steady-state test: During the steady-state test, input parameters like voltage and current remain constant. These tests are carried out with constant voltage and current inputs applied to the PMU under test. All the measurements are carried out after system stabilization to rule out any transients.

$>$ Dynamic test: During the dynamic test, one or more input parameters are varied during the test period. During these tests, the magnitude/frequency of the input signal is either modulated or ramped or step change of input signal in terms of magnitude or phase has been performed.

\section{Phasor measurement unit calibration system}

The PMU calibration system compliant with the IEEE standards facilitates PMU manufacturers to verify their PMU performance. It provides an option to the users to validate PMUs before using them on the field and allows 
Table 1. Different tests mentioned in IEEE: C37.118.1-2011

\begin{tabular}{llc}
\hline Test category & \multicolumn{1}{c}{ Test parameter } & Section in C37.118.1-2011 \\
\hline Performance class & P class/M class & 5.5 .2 \\
Reporting rate & $10 / 25 / 50$ Fs @50 Hz system & 5.4 .1 \\
& $10 / 12 / 15 / 20 / 30 / 60$ Fs @60 Hz system & 5.4 .1 \\
Steady-state compliance & Signal frequency & 5.5 .5 \\
& Signal magnitude: voltage & 5.5 .5 \\
& Single magnitude: current & 5.5 .5 \\
& Phase angle & 5.5 .5 \\
& Harmonic distortion & 5.5 .5 \\
Dynamic compliance & Out-of-band interference & 5.5 .5 \\
& Amplitude modulation & 5.5 .6 \\
& Phase modulation & 5.5 .6 \\
& Ramp of system frequency & 5.5 .7 \\
& Step changes in amplitude & 5.5 .8 \\
Reporting latency & Step change in phase & 5.5 .8 \\
\hline
\end{tabular}

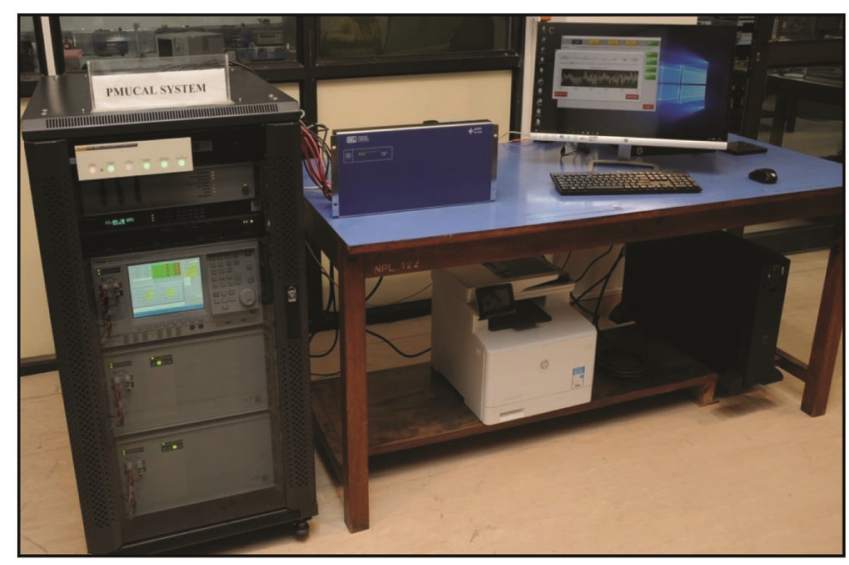

Figure 1. Traceable CSIR-NPL PMU-CAL system.

calibration at periodic intervals, thereby ensuring quality and reliability. The conventional PMU calibrator used is complex, time-consuming and manually operated, which requires a highly skilled operator for equipment operation. To perform complex measurements, operator proficiency is needed for continuous interaction in manual mode. First, the measurement set-up for PMU calibration is complex and second, the manual measurements make it further time-consuming, taking two to six weeks to complete the calibration of a PMU.

The PMU calibration cost due to manual operation, which requires a highly skilled and professional operator is very high. Besides this, the calibrator must have its traceability to the primary standards maintained by NMIs to ensure its accuracy and reliability. The recommended PMU calibrator uncertainty should be less than $10 \%$ of the limit allowed by IEEE. Due to the extensive test processing time, it is difficult for the manual PMU calibrator system to meet stringent accuracy requirements. The solution to these above-mentioned limitations is provided by an automated PMU calibration system
(PMU-CAL) with high precision and traceable to the primary standards of CSIR-NPL ${ }^{11,12}$. The CSIR-NPL PMU calibrator is an integrated measurement system that completes more than 300-500 automated tests (it includes steady-state and dynamic tests along with latency tests) within $5-6 \mathrm{~h}$ for $\mathrm{P}$ and $\mathrm{M}$ class at desired frame rates according to the IEEE C37.118.1a-2014 standard.

Power grids worldwide depend on PMUs for gathering on-line information about line frequency, current, voltage and phase that exist within the grid. This necessitates PMU calibration through a system that is traceable to ensure its data consistency with high accuracy and reliability. As shown in Figure 1, the recently established CSIR-NPL PMU-CAL system is ideal to provide a perfect solution for PMU calibration. This integrated and traceable PMU-CAL system fully complies with the IEEE C37.118.1-2011 and C37.118.1a-2014 synchrophasor standards and IEEE guide C37.242-2013 for PMU operation, installation and verification. For calibrating the PMU using this system, different voltage and current signal combinations are generated considering the actual grid working environment. The response from PMU is then recorded in automation mode using the software. Before this, the PMU is configured according to the testing environment. Figure $2 a$ provides a pictorial view of the PMU-CAL system automation software. The software also helps calculate TVE, FE and rate of change of frequency error (RFE), and the obtained values are then compared with the applied signals. Figure $2 b$ shows the real-time environment for a single test run by an automation software. The presentation of TVE is shown in Figure 3, where $V_{\text {th }}$ is the true phasor, $V_{\mathrm{ob}}$ the measured phasor and TVE is the magnitude, $\left|V_{\text {diff }}\right|$.

The PMU-Cal system can produce multiple complex signals, including harmonics and inter-harmonics. Using this system, one can generate precise voltage and current stimulus up to six digits with better than 50 ppm accuracy. The system is made up of a three-phase electrical power 


\section{RESEARCH ARTICLES}
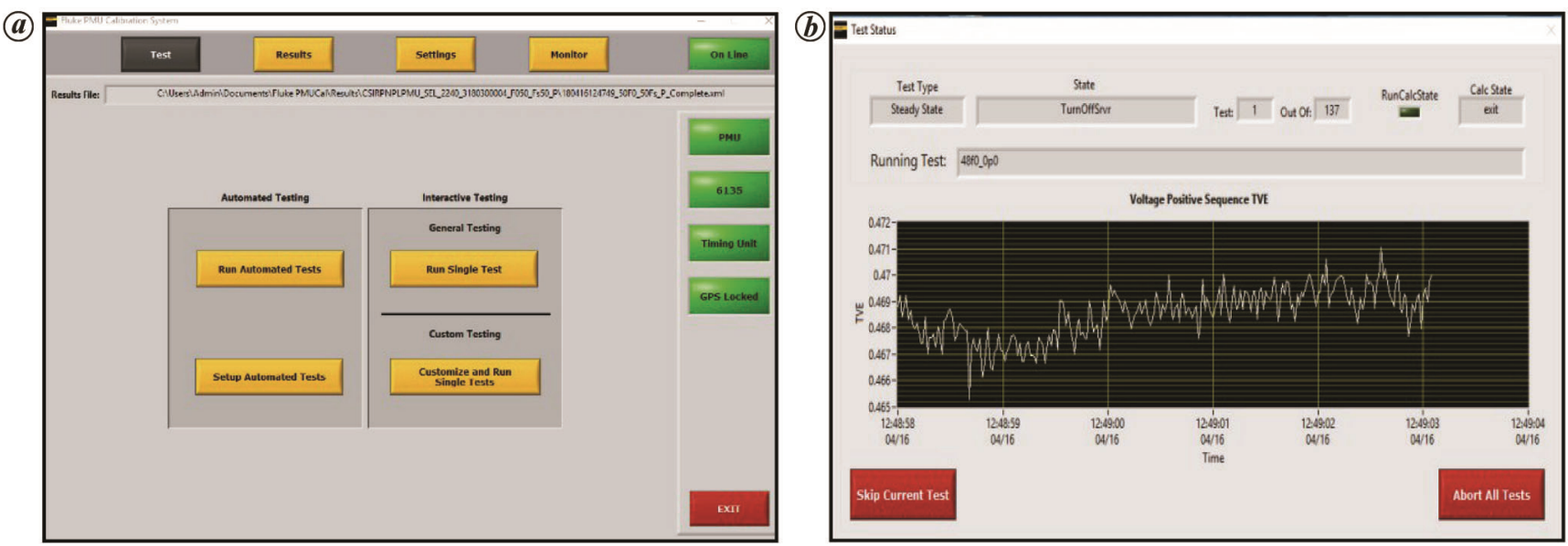

Figure 2. CSIR-NPL PMU-CAL system automation software. (a) Front panel and (b) real-time environment for a single test run.

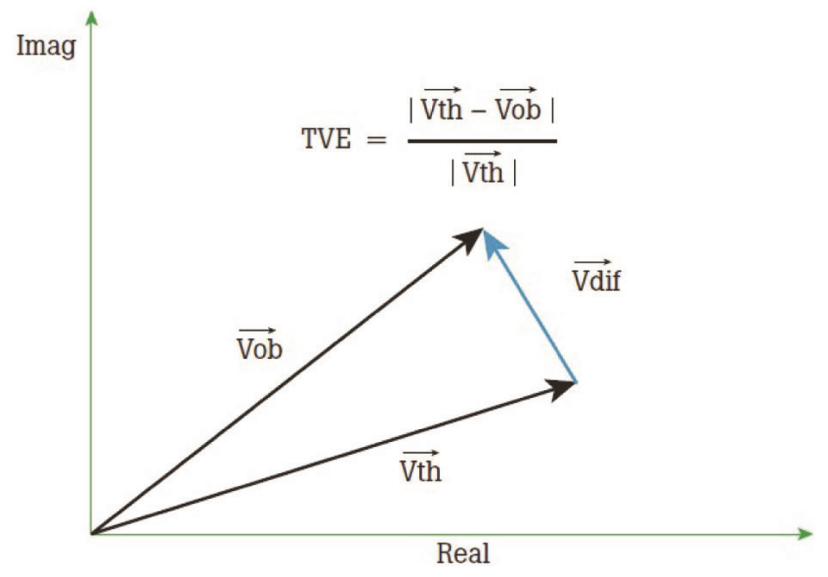

Figure 3. Total vector error (TVE).

standard, GPS receiver, system controller and system timing unit (STU) interconnected together as shown in Figure 1. The electrical power standard comprises a single master unit and dual auxiliary units. They supply a three-phase current and voltage signal to the PMU under test.

The function of the GPS receiver is to provide the PMU and PMU calibrator (PMU-CAL) system with a source of Universal Coordinated Time (UTC) for time stamping. The STU provides synchronization among the tests during calibration and accordingly controls the timing. The interface and control between the computer and the system are achieved through the system control$\operatorname{ler}^{13,14}$

Presently, CSIR-NPL has the PMU-CAL system facility with traceability to national standards for calibrating PMUs (Figure 4). The uncertainty of the system is in the range $\pm 0.005 \%- \pm 0.010 \%$. This automated system runs several dynamic and static tests that comply with the IEEE standards and completes the full calibration of a PMU in a few hours.

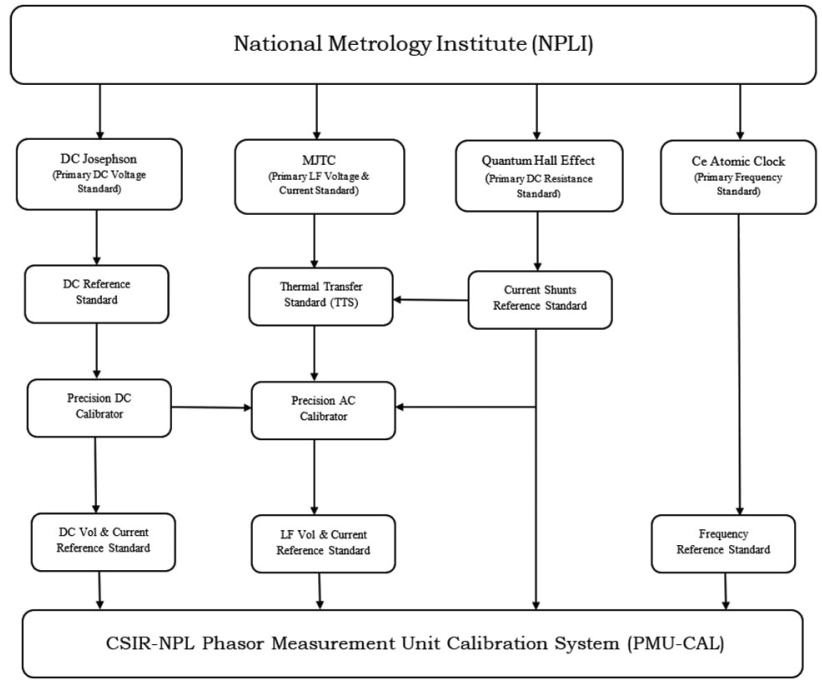

Figure 4. PMU-CAL system traceability chart.

The individual components of the PMU-CAL system and its interface with PMU under test are achieved through automation software. The software assists in PMU configuration; its testing and calibration according to IEEE standards C37.118.1-2011 and C37.118.1a-2014. Two modes of PMU configuration are available according to the needs. $\mathrm{P}$ class is the protection class having a faster time response required for the on-field protection unit. $M$ class is the measurement class, which does not require faster time response but is used for analytic measurements with greater precision.

The importance of time synchronization makes PMU one of the most important and reliable data-acquisition equipment across the grids. The time stamping is achieved using GPS-derived UTC. The GPS signal used by the PMU-CAL system has been validated using a CRO-based measurement method. The signal validation confirms that the accuracy of the GPS signal received by 
Table 2. PMU calibration results for steady-state test at $63 \mathrm{~V}$ and $1 \mathrm{~A}$ at $50 \mathrm{fps}$

\begin{tabular}{|c|c|c|c|c|c|c|}
\hline Steady test parameters & Error & $V_{\mathrm{A}}$ & $V_{\mathrm{B}}$ & $V_{\mathrm{C}}$ & $\begin{array}{l}\text { Voltage positive } \\
\text { sequence }\end{array}$ & $\begin{array}{l}\text { IEEE } \\
\text { limits }\end{array}$ \\
\hline \multicolumn{7}{|l|}{ Voltage with its measurement uncertainties } \\
\hline \multirow[t]{3}{*}{ Frequency response } & TVE $(\%)$ & 0.4472 & 0.4177 & 0.406 & 0.4192 & $1.00 \%$ \\
\hline & $\mathrm{FE}(\mathrm{Hz})$ & 0.00189 & 0.00189 & 0.00189 & 0.00189 & $0.005 \mathrm{~Hz}$ \\
\hline & RFE (Hz/s) & 0.05856 & 0.05856 & 0.05856 & 0.05856 & $0.1 \mathrm{~Hz} / \mathrm{s}$ \\
\hline \multirow[t]{3}{*}{ Signal magnitude (voltage and current) } & TVE $(\%)$ & 0.7605 & 0.6276 & 0.5314 & 0.4531 & $1.00 \%$ \\
\hline & $\mathrm{FE}(\mathrm{Hz})$ & 0.00756 & 0.00756 & 0.00756 & 0.00756 & Infinite \\
\hline & $\mathrm{RFE}(\mathrm{Hz} / \mathrm{s})$ & 0.3542 & 0.3542 & 0.3542 & 0.3542 & Infinite \\
\hline \multirow[t]{3}{*}{ Harmonic distortion (single harmonic) } & TVE $(\%)$ & 0.4439 & 0.4225 & 0.4056 & 0.4196 & $1.00 \%$ \\
\hline & $\mathrm{FE}(\mathrm{Hz})$ & 0.00095 & 0.00095 & 0.00095 & 0.00095 & $0.025 \mathrm{~Hz}$ \\
\hline & $\mathrm{RFE}(\mathrm{Hz} / \mathrm{s})$ & 0.02384 & 0.02384 & 0.02384 & 0.02384 & Infinite \\
\hline \multirow[t]{3}{*}{ Out-of-band interference (inter-harmonics) } & TVE (\%) & 0.4491 & 0.4449 & 0.4223 & 0.4318 & $1.3 \%$ \\
\hline & $\mathrm{FE}(\mathrm{Hz})$ & 0.00642 & 0.00642 & 0.00642 & 0.00642 & $0.01 \mathrm{~Hz}$ \\
\hline & $\mathrm{RFE}(\mathrm{Hz} / \mathrm{s})$ & 0.6098 & 0.6098 & 0.6098 & 0.6098 & Infinite \\
\hline Steady test parameters & Error & $I_{\mathrm{A}}$ & $I_{\mathrm{B}}$ & $I_{\mathrm{C}}$ & $\begin{array}{l}\text { Current positive } \\
\text { sequence }\end{array}$ & $\begin{array}{l}\text { IEEE } \\
\text { limits }\end{array}$ \\
\hline \multicolumn{7}{|l|}{ Current with its measurement uncertainties } \\
\hline \multirow[t]{3}{*}{ Frequency response } & TVE $(\%)$ & 0.3943 & 0.3253 & 0.4568 & 0.388 & $1.00 \%$ \\
\hline & $\mathrm{FE}(\mathrm{Hz})$ & 0.00189 & 0.00189 & 0.00189 & 0.00189 & $0.005 \mathrm{~Hz}$ \\
\hline & $\mathrm{RFE}(\mathrm{Hz} / \mathrm{s})$ & 0.05856 & 0.05856 & 0.05856 & 0.05856 & $0.1 \mathrm{~Hz} / \mathrm{s}$ \\
\hline \multirow[t]{3}{*}{ Signal magnitude (voltage and current) } & TVE $(\%)$ & 0.5945 & 0.9698 & 0.83 & 0.784 & $1.00 \%$ \\
\hline & $\mathrm{FE}(\mathrm{Hz})$ & 0.00756 & 0.00756 & 0.00756 & 0.00756 & Infinite \\
\hline & $\mathrm{RFE}(\mathrm{Hz} / \mathrm{s})$ & 0.3542 & 0.3542 & 0.3542 & 0.3542 & Infinite \\
\hline \multirow[t]{3}{*}{ Harmonic distortion (single harmonic) } & TVE $(\%)$ & 0.385 & 0.3029 & 0.4197 & 0.3633 & $1.00 \%$ \\
\hline & $\mathrm{FE}(\mathrm{Hz})$ & 0.00095 & 0.00095 & 0.00095 & 0.00095 & $0.025 \mathrm{~Hz}$ \\
\hline & $\mathrm{RFE}(\mathrm{Hz} / \mathrm{s})$ & 0.02384 & 0.02384 & 0.02384 & 0.02384 & Infinite \\
\hline \multirow[t]{3}{*}{ Out-of-band interference (inter-harmonics) } & TVE $(\%)$ & 0.3994 & 0.3165 & 0.4444 & 0.3784 & $1.3 \%$ \\
\hline & $\mathrm{FE}(\mathrm{Hz})$ & 0.00642 & 0.00642 & 0.00642 & 0.00642 & $0.01 \mathrm{~Hz}$ \\
\hline & $\mathrm{RFE}(\mathrm{Hz} / \mathrm{s})$ & 0.6098 & 0.6098 & 0.6098 & 0.6098 & Infinite \\
\hline
\end{tabular}

$V_{\mathrm{A}}, V_{\mathrm{B}}$ and $V_{\mathrm{C}}$ are voltage phase A, voltage phase B and voltage phase C with uncertainty in voltage $\pm 0.08 \%$, line frequency: $\pm 0.0001 \mathrm{~Hz}$ and GPS clock frequency: $\pm 5 \mu \mathrm{Hz}$.

$I_{\mathrm{A}}, I_{\mathrm{B}}$ and $I_{\mathrm{C}}$ are current phase A, current phase B and current phase C with uncertainty in current $\pm 0.12 \%$, line frequency: $\pm 0.0001 \mathrm{~Hz}$ and GPS clock frequency: $\pm 5 \mu \mathrm{Hz}$.

the CSIR-NPL PMU-CAL system is $0.441 \mu \mathrm{s}$ compared to the IEEE limit of less than $1 \mu \mathrm{s}$. There are different signal formats like pulse per signal (PPS), an Inter Range Instrumentation Group Time Code Format B (IRIG-B), etc. The $10 \mathrm{MHz}$ signals from the primary standard caesium atomic clock with high stability are used to generate a clock reference signal ${ }^{15,16}$.

\section{Traceability}

CSIR-NPL, an NMI of India with a mandate to maintain, develop and upgrade the national standards according to the country's needs, paves the way for disseminating the measurement traceability to the base SI units. This helps the industries, regional test and calibration laboratories, academic institutions, etc. keep their calibration and measurement capabilities up-to-date with improved uncertainty. CSIR-NPL has the relevant expertise and required infrastructure to cater to these demands and accordingly, it helps the industries and other sectors which in turn results in their overall development and improves the quality of life. The intercomparison is carried out at regular intervals with the goal of achieving international equivalence. The measurement traceability of the CSIR-NPL PMUCAL system has been established against the primary standards of the country. Figure 4 shows the traceability to national standards using the known reference standards through an unbroken chain of measurements ${ }^{17}$. For uniformity and consistency, it is essential that all PMUs that are being mounted or are under installation at the power grid are calibrated using the traceable PMU-CAL system. The objective of PMU calibrator system traceability is to ensure that all PMU measurements are accurate, reliable and fully interchangeable, and comply with the latest IEEE standards. If any PMU in use is not calibrated it may give misleading data, thereby resulting in financial loss and will be a concern for power grid safety.

As shown in Figure 4, traceability of the PMU calibrator is achieved through the following standards:

(a) Programmable Josephson voltage standard at NPLI; a primary standard of DC voltage. 

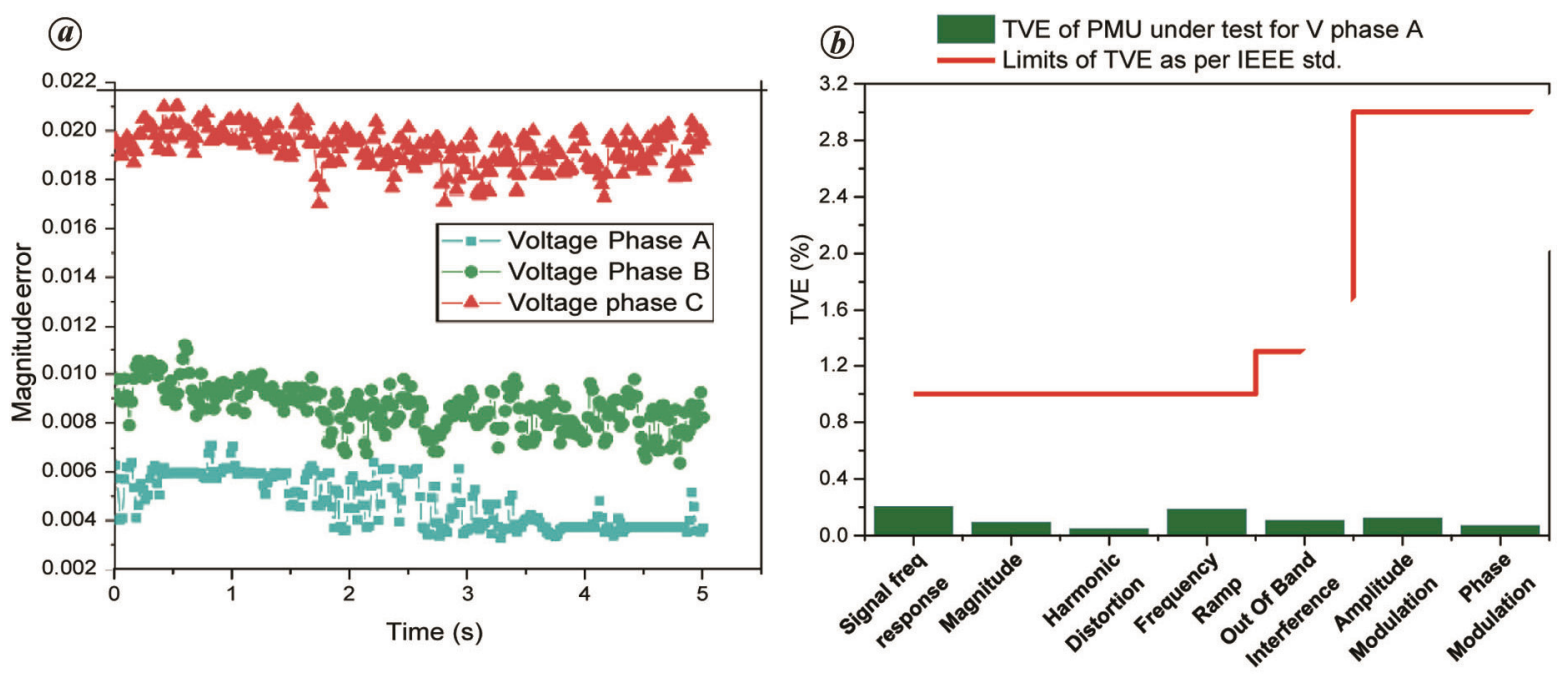

Figure 5. PMU measurement results: $\boldsymbol{a}$, Voltage magnitude error for all three phases; $\boldsymbol{b}$, TVE of all the voltage phases shown as histogram along with the IEEE limits as a line.

(b) Quantum hall resistance standard at NPLI; a primary standard of DC resistance.

(c) Multi junction thermal converter (MJTC) at NPLI; a primary standard of LF voltage.

(d) Caesium atomic clocks at NPLI; a primary standard of frequency.

(e) MJTC with current shunts at NPLI; a primary standard of LC current.

These primary standards are maintained at CSIR-NPL and are used on a periodic basis to calibrate the CSIR-NPL PMU calibrator with an uncertainty of $\pm(50-100) \mathrm{ppm}$.

\section{Results and discussion}

PMUs play an important role in observing and enhancing the stability of the power grid. To maintain the efficiency of the power grid, PMUs need to be highly accurate, reliable and repeatable, which is only possible by performing comprehensive and traceable PMU calibration using the PMU calibrator. PMUs are being placed strategically at national power grids to analyse their performance in realtime using GPS-based UTC. The CSIR-NPL PMUCAL system has been used to calibrate a reference standard PMU according to IEEE C37.118.1-2011 and C37.118.1a-2014 standards. The complete test for $\mathrm{M}$ class configured PMU has been done at $63 \mathrm{~V}$ and $1 \mathrm{~A}$ at $50 \mathrm{fps}$ (frame per second), which takes around $6-7 \mathrm{~h}$ to complete in automatic mode, once configured. It includes 283 individual tests under static and dynamic conditions according to IEEE standards. Table 2 shows the results obtained for three voltage phases and current phases, and the limits defined in the standards. The magnitude error in voltage for all three phases is shown in Figure $5 a$, whereas the PMU (TVE) for all the concerned individual tests is shown in Figure $5 b$ as a histogram along with the IEEE limits displayed in the form of line. The test result clearly indicates that the difference in magnitude error among all the three voltage phases is negligible compared to the allowed permissible limits of IEEE standards. TVE for the tests carried out is well below the IEEE limits. This shows that the validation of the PMU has been successfully completed according to IEEE standard limits, and it can be safely deployed at any point of the power grid.

\section{Conclusion}

Calibrated PMUs will be an essential and reliable tool to provide more intelligent monitoring, control and protection for the national power grid. The PMU-CAL system has been successfully installed and commissioned at CSIR-NPL, whose traceability has been established against the respective national standards. The time taken to calibrate a PMU has been reduced to a few hours, in contrast to the traditional technique that takes more than a week with skilled manpower. PMU data without having traceability are creating perceptions which are far from reality. We urge the policymakers and regulators to ensure that all PMUs should be technically evaluated from a traceable PMU-CAL system before their deployment. They should also be calibrated periodically to avoid any power outages and cascading blackouts, minimizing economic losses to the country. With the traceable measurements, we expect that all the PMUs deployed in the national power grid system after calibration will help in obtaining accurate and uniform data. This will increase efficiency, improve reliability and enhance the quality of the power grid. 
1. North American Synchrophasor Initiative; http://www.naspi.org

2. Zhang, P., Phasor Measurement Unit (PMU) Implementation and Applications, EPRI Report No. 1015511, Electric Power Research Institute, Palo Alto, California, 31 October 2007, p. 2-17; http://www.naspi.org/repository/project details.aspx?pid=116

3. NERC, NERC Real-Time Application of Synchrophasors for Improving Reliability, North American Electric Reliability Council, Princeton, New Jersey, 19 October 2010, p. 77.

4. Staffs of the Federal Energy Regulatory Commission and North American Electric Reliability Corporation; Arizona-Southern California Outages on September 8, 2011, Causes and Recommendations, 27 April 2012

5. IEEE Standards Association, Power and Energy Society, Power Systems Relaying Committee, IEEE C37.118.1, Standard for Synchrophasor Measurements for Power Systems.

6. IEEE Standards Association, Power and Energy Society, Power Systems Relaying Committee, IEEE C37.242, Guide for Synchronization, Calibration, Testing, and Installation of Phasor Measurement Units (PMU) for Power System Protection and Control.

7. IEEE Standard for Synchrophasors for Power Systems. IEEE C37.118-2005.

8. IEEE Standard for Synchrophasors Measurement for Power Systems. IEEE C37.118.1-2011.

9. IEEE Standard for Synchrophasors Measurement for Power Systems, Amendment 1: Modification of Selected Performance Requirements, IEEE C37.118.1a-2014.

10. IEEE Guide for Synchronization, Calibration, Testing, and Installation of Phasor Measurement Units (PMUs) for Power Systems Protection and Control, IEEE C37.242-2013.

11. Pirret, R., New standards for test and calibration of phasor measurement units. In NCSL International Workshop and Symposium, Sacramento, California, USA, 2012, pp. 1-12.
12. Fernandez, J. O., The Virginia Tech Calibration System, 2011, pp. $1-30$.

13. Narendra, K. et al., Calibration and testing of TESLA phasor measurement unit (PMU) using DOBLE F6150 test instrument, Bulk Power System Dynamics and Control-VII. Revitalizing Operational Reliability. In iREP Symposium, Charleston, SC, USA, 2007, pp. $1-13$.

14. Lin, Z. Z. et al., Dynamic performance test of single-phase phasor measurement units. In Power and Energy Society General Meeting, Michigan, USA, 2011, pp. 1-3.

15. Komarnicki, P. et al., Practical experience with PMU system testing and calibration requirements. In Power and Energy Society General Meeting - Conversion and Delivery of Electrical Energy in the 21st Century, IEEE, 2008, pp. 1-5.

16. Fluke 6135A/PMU Calibration System Operators Manual; http:/us.flukecal.com/products/electrical-calibration/electricalcalibrators/6135apmucal-phasor- measurement-unit-calibration

17. Tang, Y. and Stenbakken, G. N., Traceability of calibration for phasor measurement unit. In Power and Energy Society General Meeting, San Diego, California, USA, 2012, pp. 1-5.

ACKNOWLEDGEMENTS. We thank Bill Spath (Fluke Calibration, USA), Amiya Biswal (Fluke Technologies Pvt Ltd, New Delhi) and P. S. Negi, Archana Sahu, Swati Kumari and Sunidhi Luthra (CSIR-NPL, New Delhi) for their valuable contributions and technical discussions during this study. We also thank Amreek Singh (CSIR-NPL) for technical help and logistic support, and CSIR-NPL for financial assistance.

Received 5 November 2019; revised accepted 5 April 2021

doi: $10.18520 / \mathrm{cs} / \mathrm{v} 121 / \mathrm{i} 2 / 248-254$ 